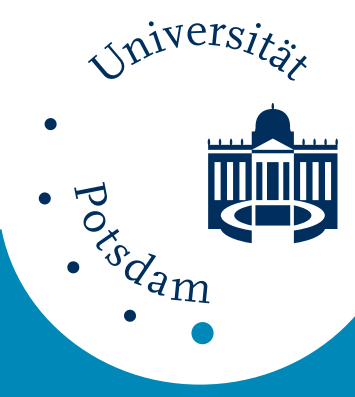

Universität Potsdam

Fred Feudel, Norbert Seehafer, Barak Galanti, Sten Rüdiger

Symmetry breaking bifurcations for the magnetohydrodynamic equations with helical forcing

NLD Preprints ; 31 


\title{
Symmetry breaking bifurcations for the magnetohydrodynamic equations with helical forcing
}

\author{
F. Feudel, ${ }^{1}$ N. Seehafer, ${ }^{1}$ B. Galanti, ${ }^{2}$ and S. Rüdiger ${ }^{1}$ \\ ${ }^{1}$ Max-Planck-Gruppe Nichtlineare Dynamik, Universität Potsdam, PF 601553, D-14415 Potsdam, Germany \\ ${ }^{2}$ Department of Chemical Physics, Weizmann Institute of Science, Rehovot 76100, Israel
}

\begin{abstract}
We have studied the bifurcations in a three-dimensional incompressible magnetofluid with periodic boundary conditions and an external forcing of the ABC type. Bifurcation-analysis techniques have been applied to explore the qualitative behavior of solution branches. Due to the symmetry of the forcing, the equations are equivariant with respect to a group of transformations isomorphic to the octahedral group, and we have paid special attention to symmetry breaking effects. As the Reynolds number is increased, the primary non-magnetic steady state, the $\mathrm{ABC}$ flow, loses its stability to a periodic magnetic state, showing the appearance of a generic dynamo effect; the critical value of the Reynolds number for the instability of the ABC flow is decreased compared to the purely hydrodynamic case. The bifurcating magnetic branch in turn is subject to secondary, symmetry breaking bifurcations. We have traced periodic and quasiperiodic branches until they end up in chaotic states. In particular detail we have analyzed the subgroup symmetries of the bifurcating periodic branches, which are closely related to the spatial structure of the magnetic field.
\end{abstract}

47.20.Ky, 47.65.+a, 47.27.Cn, 95.30.Qd

\section{INTRODUCTION}

The generation and maintenance of magnetic fields by the motion of electrically conducting fluids is the subject of dynamo theory. One of its main objectives is to explain the existence of long lasting cosmical magnetic fields, as, for example, those of the Earth and the Sun. For a recent account of dynamo theory we refer to Ref. [1].

Usually the magnetohydrodynamic (MHD) equations are employed to describe the dynamo effect. When the magnetic energy is small compared to that of the velocity field, one can consider the kinematic problem, that is, the induction equation for a prescribed velocity field disregarding the response to the motion of the fluid. In the kinematic frame the question is, whether a fluid motion can amplify and maintain weak seed of magnetic field. One of the successful examples to produce a dynamo effect are the ABC flows (named after Arnold, Beltrami and Childress), firstly investigated by Arnold [2],

$$
\begin{aligned}
\boldsymbol{v}_{\mathrm{ABC}}= & \left(A \sin k_{0} z+C \cos k_{0} y, B \sin k_{0} x+A \cos k_{0} z,\right. \\
& \left.C \sin k_{0} y+B \cos k_{0} x\right),
\end{aligned}
$$

where $A, B, C$ denote constant coefficients and $k_{0}$ is a (also constant) positive wave number. They are a strongly helical flows satisfying the Beltrami condition, $\boldsymbol{\nabla} \times \boldsymbol{v}=\lambda \boldsymbol{v}$, with $\lambda=k_{0}$, a necessary condition for the existence of chaotic domains in the flow [2]. For these reasons, they have received much interests [3,4], notably in the kinematic context as candidates for fast dynamos $[5,6]$ (for which the growth rate remains bounded from below by a positive constant as the magnetic diffusivity tends to zero).

The $\mathrm{ABC}$ flows are steady solutions of the incompressible Euler equation. They are also steady solutions of the incompressible Navier-Stokes equation (NSE) [Eq. (3) below with the magnetic field, $\boldsymbol{B}$, dropped] if an external body force

$$
\boldsymbol{f}=-\nu \nabla^{2} \boldsymbol{v}_{\mathrm{ABC}}=\nu k_{0}^{2} \boldsymbol{v}_{\mathrm{ABC}}
$$

is applied in order to compensate for viscous losses. But they are only stable solutions below a critical strength of the forcing or critical Reynolds number, respectively - investigations of their linear stability are due to Galloway and Frisch [7]. The nonlinear behavior of solutions to the NSE with the forcing given by Eq. (2) has been studied numerically by Zheligovsky and Pouquet [8] and by Podvigina and Pouquet [9]. These authors report various bifurcations that occur as the strength of forcing (or the Reynolds number) is raised and that lead to qualitatively different and partially coexisting solution branches, including chaotic ones.

Imposing the same kind of forcing, Galanti et al. [10] investigated the complete system of MHD equations by means of numerical simulations. From kinematic studies it is known that for small Reynolds number the ABC flow with no magnetic field is also a stable solution of the MHD equations. Galanti et al. found that at some critical value of the Reynolds number, the $\mathrm{ABC}$ flow loses stability to time periodic solutions with a magnetic field, indicating the occurrence of a dynamo effect.

Continuing these studies by Galanti et al. [10] we have investigated the MHD equations with the imposed $\mathrm{ABC}$ forcing, for the special case of $A=B=C$ and $k_{0}=1$, by applying methods of the numerical bifurcation analysis as well as group-theoretical methods. The aim of the present paper is to describe the bifurcation structure in dependence on the Reynolds number as control parameter. The MHD equations with our special forcing are equivariant with respect to a group of transformations isomorphic to the octahedral group. We have paid special attention to symmetry breaking effects of the bifur- 
cations. In particular, we have determined the subsymmetries of bifurcating solution branches. In a companion paper [11] a generalized ABC forcing is applied to investigate the influence of the degree of helicity in the forcing on the character of the first bifurcation of the primary non-magnetic stationary state.

In Sec. II we cast the three-dimensional MHD equations into spectral form and explain the kind of truncation used, while in Sec. III the external forcing and its associated symmetries are explained. In Sec. IV we present the results of our numerical bifurcation analysis, i.e., the bifurcations and their symmetry breaking effects. Then in Sec. V, the spatio-temporal structure of the magnetic field as well as its symmetry properties are considered. Sec. VI, finally, contains a brief discussion.

\section{BASIC EQUATIONS AND TRUNCATION}

We start from the equations for a non-relativistic, incompressible, electrically conducting fluid with constant material properties (cf., e.g., Roberts [12]),

$$
\begin{aligned}
& \rho\left[\frac{\partial \boldsymbol{v}}{\partial t}+(\boldsymbol{v} \cdot \nabla) \boldsymbol{v}\right]=\rho \nu \nabla^{2} \boldsymbol{v}-\nabla p-\frac{1}{2 \mu_{0}} \nabla \boldsymbol{B}^{2} \\
& +\frac{1}{\mu_{0}}(\boldsymbol{B} \cdot \boldsymbol{\nabla}) \boldsymbol{B}+\boldsymbol{f} \\
& \frac{\partial \boldsymbol{B}}{\partial t}+(\boldsymbol{v} \cdot \boldsymbol{\nabla}) \boldsymbol{B}=\eta \nabla^{2} \boldsymbol{B}+(\boldsymbol{B} \cdot \boldsymbol{\nabla}) \boldsymbol{v} \\
& \boldsymbol{\nabla} \cdot \boldsymbol{v}=0, \quad \boldsymbol{\nabla} \cdot \boldsymbol{B}=0,
\end{aligned}
$$

where $\rho$ is the mass density, $p$ the thermal pressure, $\nu$ the kinematic viscosity, $\mu_{0}$ the magnetic permeability in a vacuum, $\eta$ the magnetic diffusivity $\left(\eta=\left(\mu_{0} \sigma\right)^{-1}, \sigma\right.$ denoting the electrical conductivity), and $\boldsymbol{f}$ an external body force. The third and fourth terms on the right-hand side of Eq. (3) constitute the Lorentz force. Transforming to non-dimensional quantities according to

$$
\begin{array}{r}
\boldsymbol{x} \rightarrow \boldsymbol{x} / L, \quad t \rightarrow t / \frac{L^{2}}{\nu}, \quad \boldsymbol{v} \rightarrow \boldsymbol{v} / \frac{\nu}{L}, \\
p \rightarrow p / \frac{\rho \nu^{2}}{L^{2}}, \quad \boldsymbol{f} \rightarrow \boldsymbol{f} / \frac{\rho \nu^{2}}{L^{3}}, \quad \boldsymbol{B} \rightarrow \boldsymbol{B} / \frac{\nu}{L} \sqrt{\mu_{0} \rho}
\end{array}
$$

Eqs. (3)-(4) become

$$
\frac{\partial \boldsymbol{v}}{\partial t}+(\boldsymbol{v} \cdot \nabla) \boldsymbol{v}=\nabla^{2} \boldsymbol{v}-\nabla p-\frac{1}{2} \nabla \boldsymbol{B}^{2}+(\boldsymbol{B} \cdot \boldsymbol{\nabla}) \boldsymbol{B}+\boldsymbol{f}
$$

$$
\frac{\partial \boldsymbol{B}}{\partial t}+(\boldsymbol{v} \cdot \nabla) \boldsymbol{B}=P_{\mathrm{m}}^{-1} \nabla^{2} \boldsymbol{B}+(\boldsymbol{B} \cdot \boldsymbol{\nabla}) \boldsymbol{v}
$$

where $P_{\mathrm{m}}$ is the magnetic Prandtl number, $P_{\mathrm{m}}=\nu / \eta$.
We impose periodic boundary conditions and consider the equations in the domain $\Omega=[2 \pi \times 2 \pi \times 2 \pi]$, where the mean values of $\boldsymbol{v}, \boldsymbol{B}, p$ and consequently of $\boldsymbol{f}$ are assumed to vanish. The solutions can be expanded into to the complete set of orthogonal eigenfunctions of the Stokes operator,

$$
\begin{gathered}
\boldsymbol{v}(\boldsymbol{x})=\sum_{\substack{\boldsymbol{k} \in \mathbb{Z}^{3} \\
\boldsymbol{k} \neq \mathbf{0}}}\left(v_{\boldsymbol{k}}^{(1)} \boldsymbol{e}_{\boldsymbol{k}}^{(1)}+v_{\boldsymbol{k}}^{(2)} \boldsymbol{e}_{\boldsymbol{k}}^{(2)}\right) \exp (\mathrm{i} \boldsymbol{k} \cdot \boldsymbol{x}), \\
\boldsymbol{B}(\boldsymbol{x})=\sum_{\substack{\boldsymbol{k} \in \mathbb{Z}^{3} \\
\boldsymbol{k} \neq 0}}\left(B_{\boldsymbol{k}}^{(1)} \boldsymbol{e}_{\boldsymbol{k}}^{(1)}+B_{\boldsymbol{k}}^{(2)} \boldsymbol{e}_{\boldsymbol{k}}^{(2)}\right) \exp (\mathrm{i} \boldsymbol{k} \cdot \boldsymbol{x}), \\
\boldsymbol{f}(\boldsymbol{x})=\sum_{\substack{\boldsymbol{k} \in \mathbb{Z}^{3} \\
\boldsymbol{k} \neq 0}}\left(f_{\boldsymbol{k}}^{(1)} \boldsymbol{e}_{\boldsymbol{k}}^{(1)}+f_{\boldsymbol{k}}^{(2)} \boldsymbol{e}_{\boldsymbol{k}}^{(2)}\right) \exp (\mathrm{i} \boldsymbol{k} \cdot \boldsymbol{x}), \\
p(\boldsymbol{x})=\sum_{\boldsymbol{k} \in \mathbb{Z}^{3}} p_{\boldsymbol{k}} \exp (\mathrm{i} \boldsymbol{k} \cdot \boldsymbol{x}),
\end{gathered}
$$

where we have used polarization vectors $\boldsymbol{e}_{\boldsymbol{k}}^{(1)}, \boldsymbol{e}_{\boldsymbol{k}}^{(2)}$ perpendicular to $\boldsymbol{k}$,

$$
\begin{gathered}
\boldsymbol{e}_{\boldsymbol{k}}^{(i)} \cdot \boldsymbol{k}=0, \boldsymbol{e}_{\boldsymbol{k}}^{(1)} \cdot \boldsymbol{e}_{\boldsymbol{k}}^{(2)}=0, \boldsymbol{e}_{\boldsymbol{k}}^{(i)} \cdot \boldsymbol{e}_{\boldsymbol{k}}^{(i)}=1, \quad \boldsymbol{e}_{-\boldsymbol{k}}^{(i)}=\boldsymbol{e}_{\boldsymbol{k}}^{(i)} \\
\text { for } i=1,2,
\end{gathered}
$$

such that Eq. (5) is satisfied automatically. Because of the last condition in Eq. (13) we have

$$
v_{-\boldsymbol{k}}^{i}=v_{\boldsymbol{k}}^{i *}, \quad B_{-\boldsymbol{k}}^{i}=B_{\boldsymbol{k}}^{i *}
$$

(an asterisk indicates the complex conjugate). By means of the above Fourier ansatz, Eqs. (9)-(12), we easily get rid of both the thermal, $\nabla p$, and magnetic, $\boldsymbol{\nabla} \boldsymbol{B}^{2} / 2$, pressure terms in Eq. (3) and arrive at the following system of ordinary differential equations (ODE):

$$
\begin{aligned}
& \frac{\mathrm{d} v_{\boldsymbol{k}}^{(j)}}{\mathrm{d} t}=-\boldsymbol{k}^{2} v_{\boldsymbol{k}}^{(j)} \\
& -\mathrm{i} \sum_{\substack{\boldsymbol{p} \in \mathbb{Z}^{3} \\
\boldsymbol{p} \neq \mathbf{0}, \boldsymbol{k}}} \sum_{\alpha, \beta=1}^{2}\left(\boldsymbol{e}_{\boldsymbol{p}}^{(\alpha)} \cdot \boldsymbol{e}_{\boldsymbol{k}}^{(j)}\right)\left(\boldsymbol{e}_{\boldsymbol{k}-\boldsymbol{p}}^{(\beta)} \cdot \boldsymbol{k}\right)\left[v_{\boldsymbol{p}}^{(\alpha)} v_{\boldsymbol{k}-\boldsymbol{p}}^{(\beta)}\right. \\
& \left.-B_{\boldsymbol{p}}^{(\alpha)} B_{\boldsymbol{k}-\boldsymbol{p}}^{(\beta)}\right]+f_{\boldsymbol{k}}^{(j)}, \\
& \frac{\mathrm{d} B_{\boldsymbol{k}}^{(j)}}{\mathrm{d} t}=-P_{\mathrm{m}}^{-1} \boldsymbol{k}^{2} B_{\boldsymbol{k}}^{(j)} \\
& -\mathrm{i} \sum_{\substack{\boldsymbol{p} \in \mathbb{Z}^{3} \\
\boldsymbol{p} \neq \mathbf{0} \boldsymbol{k}}} \sum_{\alpha, \beta=1}^{2}\left(\boldsymbol{e}_{\boldsymbol{p}}^{(\alpha)} \cdot \boldsymbol{e}_{\boldsymbol{k}}^{(j)}\right)\left(\boldsymbol{e}_{\boldsymbol{k}-\boldsymbol{p}}^{(\beta)} \cdot \boldsymbol{k}\right)\left[B_{\boldsymbol{p}}^{(\alpha)} v_{\boldsymbol{k}-\boldsymbol{p}}^{(\beta)}\right. \\
& \left.-v_{\boldsymbol{p}}^{(\alpha)} B_{\boldsymbol{k}-\boldsymbol{p}}^{(\beta)}\right] .
\end{aligned}
$$

In the numerical calculations we have applied an isotropic truncation in wave number space and taken into account wavenumbers $\boldsymbol{k}$ with $\boldsymbol{k}^{2} \leq 12$. This corresponds to 89 k-vectors, which amounts to studying a system of 712 ODE. 


\section{FORCING AND SYMMETRY}

In order to compensate for viscous and ohmic losses, some kind of external forcing has to be applied in Eq. (3). We have used the (non-dimensional) forcing

$$
\boldsymbol{f}=k_{0}^{2} \boldsymbol{v}_{\mathrm{ABC}},
$$

$\boldsymbol{v}_{\mathrm{ABC}}$ defined by Eq. (1), with the restriction

$$
A=B=C=R, \quad k_{0}=1 \text {. }
$$

In the following the number $R$ introduced here will be referred to as the Reynolds number (in accordance with the definition of the Reynolds number used in previous studies $[10,9])$. In the numerical calculations we have restricted ourselves to the case $P_{\mathrm{m}}=1$ and $R$ has been our bifurcation parameter.

For this special ABC forcing the MHD equations are equivariant with respect to a discrete transformation group which contains 24 elements and is isomorphic to the octahedral group $O$ (the rotation group of a cube) $[2,13,4]$. For details of the group, namely, a list of the coordinate transformations, comments on the general group structure as well as an explicit group table, we must refer to the Appendix. The whole symmetry group is, for instance, generated by the two transformations $T_{2}$ and $T_{5}$ (see in the Appendix). A number of bifurcations observed in the system are related to the symmetries.

Each transformation $\mathrm{T}$ is a combination of a rigid rotation with a translation and can be written as

$$
\boldsymbol{x}^{\prime}=\mathrm{T} \boldsymbol{x}=\mathrm{D} \boldsymbol{x}+\boldsymbol{a},
$$

where a prime denotes transformed quantities, D is an orthogonal $3 \times 3$ matrix and $\boldsymbol{a}$ a constant vector. Associated with this transformation of the position vector $\boldsymbol{x}$ is a transformation of the vector field $\boldsymbol{v}(\boldsymbol{x})(\boldsymbol{B}(\boldsymbol{x})$ is transformed in the same way) according to

$$
\boldsymbol{v}^{\prime}(\boldsymbol{x})=\mathrm{D} \boldsymbol{v}\left(\mathrm{T}^{-1} \boldsymbol{x}\right) .
$$

In Fourier space Eq. (20) takes the form

$$
\boldsymbol{v}_{\boldsymbol{k}}^{\prime}=\mathrm{D} \boldsymbol{v}_{\tilde{\boldsymbol{k}}} \cdot \exp (-\mathrm{i} \boldsymbol{k} \cdot \boldsymbol{a})
$$

where

$$
\tilde{\boldsymbol{k}}=\mathrm{D}^{-1} \boldsymbol{k}
$$

and for the quantities $v_{\boldsymbol{k}}^{(1)}$ and $v_{\boldsymbol{k}}^{(2)}$ one obtains

$$
v_{\boldsymbol{k}}^{\prime(j)}=\exp (-\mathrm{i} \boldsymbol{k} \cdot \boldsymbol{a}) \sum_{\alpha=1}^{2} v_{\tilde{\boldsymbol{k}}}^{(\alpha)}\left(\mathrm{D} \boldsymbol{e}_{\tilde{\boldsymbol{k}}}^{(\alpha)}\right) \cdot \boldsymbol{e}_{\boldsymbol{k}}^{(j)} .
$$

By using this relation (and the corresponding one for the magnetic field) it can be checked numerically with respect to which transformations of the symmetry group, if any, a particular solution is symmetric.

\section{BIFURCATION STRUCTURE}

Table I gives an overview of the detected stablesolution branches and their symmetries. For small Reynolds numbers (weak forcing) only the directly forced modes (with $|\boldsymbol{k}|=1$ ) are excited and the pure ABC flow with vanishing magnetic field is a stable stationary solution. In our truncation (with $89 \boldsymbol{k}$ vectors), the ABC flow loses stability at $R=5.7$ and a stable periodic solution with a non-vanishing magnetic field is born. Since only a single pair of complex-conjugate eigenvalues crosses the imaginary axis at this Hopf point, the new periodic branch, Per-1, retains the full symmetry. More precisely, the solution is no longer point symmetric with respect to all symmetry transformations; under the action of some of them a time shift is produced, but the periodic orbit as a whole is invariant. In this special case the time shift can be interpreted as the action of the reflection group $Z_{2}$ mapping the orbit onto itself. In our numerical calculations we mainly examined the symmetry properties of the orbits as a whole, not also the time shifts in all details, and thus Table I refers only to the total symmetry of the orbits.

TABLE I. Overview of the different solution branches.

\begin{tabular}{llc}
\hline \hline Branch & $\begin{array}{l}\text { Interval of } \\
\text { stability for } R\end{array}$ & Symmetry \\
\hline ABC flow & $0<R<5.7$ & $O$ \\
Per-1 & $5.7<R<11.5$ & $O$ \\
Per-3 & $7.7<R<16.0$ & $D_{4}$ \\
Per-4 & $11.5<R<17.3$ & $D_{3}$ \\
Torus-3 & $16.0<R<20.0$ & \\
Torus-4 & $17.3<R<17.9$ & \\
Chaos-3 & $R \geq 20.0$ & \\
Chaos-4 & $R \geq 17.9$ & \\
\hline \hline
\end{tabular}


The numbers in the branch designations in Table I indicate the multiplicity of the branches, i.e. the number of coexisting branches that can be transformed into each other by elements of $O$. So, for instance, Per- 3 stands for three coexisting branches.

For $R=7.8$ a new (three-fold) periodic branch (Per-3) appears. This branch is from its appearance only partially symmetric. Each orbit is invariant with respect to one of the three conjugate subgroups (cf. Appendix)

$$
\begin{aligned}
& D_{4}^{1}=\left\{T_{1}, T_{2}, T_{3}, T_{4}, T_{6}, T_{22}, T_{9}, T_{20}\right\} \\
& D_{4}^{2}=\left\{T_{1}, T_{5}, T_{6}, T_{7}, T_{3}, T_{24}, T_{9}, T_{23}\right\} \\
& D_{4}^{3}=\left\{T_{1}, T_{8}, T_{9}, T_{10}, T_{3}, T_{19}, T_{6}, T_{21}\right\} .
\end{aligned}
$$

These subgroups are isomorphic to the dihedral group $D_{4}$ (the rotations and reflections of a square in a plane which leave the square invariant). Namely, the first four elements correspond to rotations about one coordinate axis and form a subgroup isomorphic to the cyclic group $Z_{4}$, while the remaining elements correspond to the four reflections of the square perpendicular to the rotational axis of the first four elements. In the octahedral group $O$ (rotation group of the cubc), which does not contain reflections, these last four elements actually correspond to rotations by $180^{\circ}$ about axes lying in the plane of the square.

When traced backwards (i.e., for decreasing Reynolds number), the branches Per-3 disappear for $R=$ 7.7, probably due to saddle-node bifurcations (turning points) for the periodic solutions. For increasing values of the Reynolds number, the branches Per -3 undergo secondary Hopf bifurcations at $R=16.0$, generating the torus branches Torus-3. Fig. 1 shows a corresponding torus solution, namely, the projection of a trajectory onto a plane spanned by one velocity and one magnetic field (Fourier) component.



FIG. 1. Torus solution for $R=16$ (branch Torus-3).
At $R=20$ these torus branches lose stability to chaotic states (see Fig. 2).

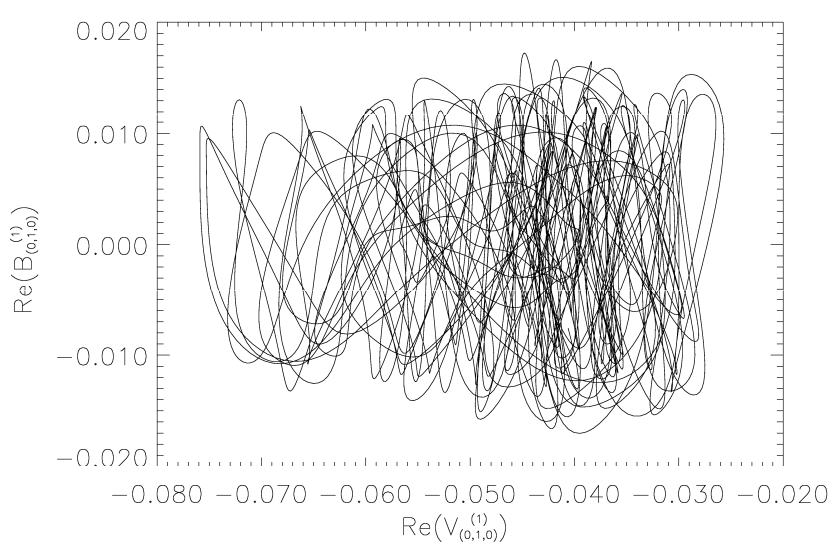

FIG. 2. Trajectory on chaotic attractor for $R=20$ (branch Chaos-3).

The chaoticity of the solutions has been verified by calculating the largest Lyapunov exponents for selected values of the bifurcation parameter using an algorithm by Shimada and Nagashima [14]. For instance, Fig. 3 shows for the branch Chaos- 3 at $R=20.0$ the cumulative value of the five largest Lyapunov exponents in dependence on the integration time.

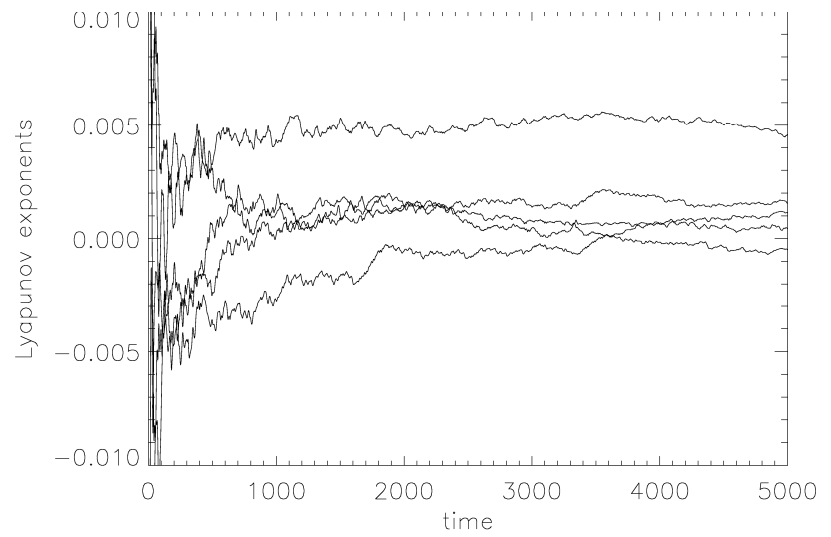

FIG. 3. The five largest Lyapunov exponents versus integration time for the branch Chaos-3 at $R=20$.

It demonstrates a good convergence, as well as that at least one of the exponents is positive.

We now return to the periodic branch Per-1, which in a symmetry breaking bifurcation at $R=11.5$ splits up into four periodic solutions forming the new branches Per-4. The symmetry breaking bifurcation retains the invariance of the solutions with respect to the following conjugated subgroups, each of which is isomorphic to the dihedral group $D_{3}$ : 


$$
\begin{aligned}
& D_{3}^{1}=\left\{T_{1}, T_{11}, T_{12}, T_{22}, T_{19}, T_{24}\right\} \\
& D_{3}^{2}=\left\{T_{1}, T_{13}, T_{14}, T_{20}, T_{24}, T_{21}\right\} \\
& D_{3}^{3}=\left\{T_{1}, T_{16}, T_{15}, T_{22}, T_{21}, T_{23}\right\} \\
& D_{3}^{4}=\left\{T_{1}, T_{18}, T_{17}, T_{20}, T_{23}, T_{19}\right\}
\end{aligned}
$$

The branches Per-4 bifurcate to the torus branches Torus-4, which are stable only for a relatively small interval of the Reynolds number, with a final transition to chaos at $R=17.9$ (generation of branches Chaos4). For a certain interval of the Reynolds number these chaotic solutions exist simultaneously with the torus branch Torus -3 .

An overview of the whole bifurcation structure is depicted schematically in Fig. 4.

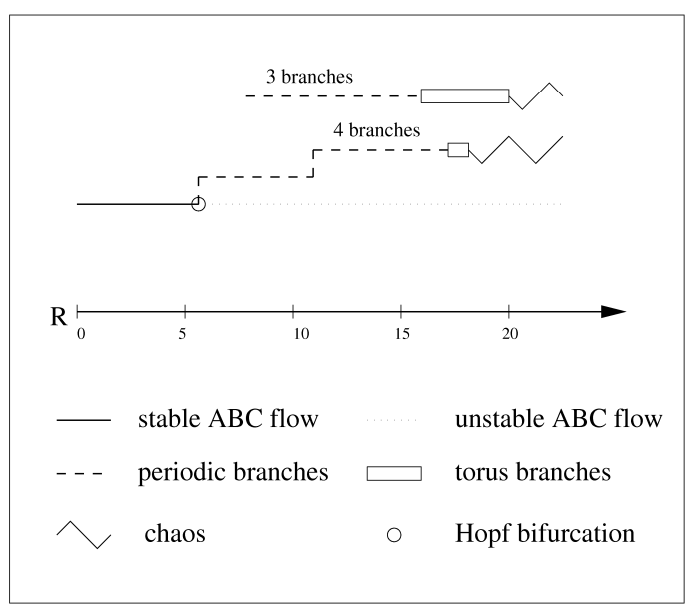

FIG. 4. Schematic bifurcation diagram

To test the sensitivity with respect to the degree of truncation, we calculated the critical Reynolds number of the first Hopf bifurcation for different truncations. There is a tendency to higher values of the critical Reynolds number for weaker truncation (more modes taken into account); a more detailed description for the dependence on the number of included modes is given in Ref. [15]. For comparison, we have also made calculations by means of a pseudospectral code, with the (compared to the spherical truncation) relatively high resolution of $16^{3}$ grid points in real space. In these calculations the first bifurcation was observed at $R=8.9$. This value coincides with the critical Reynolds number for the magnetic instability in the corresponding kinematic dynamo problem [5].

We have restricted ourselves to the forcing of the largest modes, $k_{0}=1$ [cf. Eq. (2)], and have observed an instability of the $\mathrm{ABC}$ flow on this largest scale. It should be noted that a forcing of higher modes, $k_{0}>1$, reduces the critical value of the Reynolds number by an order of magnitude which has been demonstrated for the MHD equations by Galanti et al. [10] and for the purely hydrodynamic case by Wirth et al. [16]. For instance, Wirth et al. have found a negative-viscosity large-scale instability at $R=1.92$.
Zheligovsky and Pouquet [8] and Podvigina and Pouquet [9] studied a related problem, namely, the purely hydrodynamic instabilities of the ABC forced NSE (without magnetic field). They described bifurcations, different stable-solution branches and symmetry breaking effects. Especially, they determined the value of the Reynolds number where the primary $\mathrm{ABC}$ flow (for the case $A=B=C$ ) loses its stability: $R \approx 13.044$. Since for the MHD equations already for a smaller value of $R$ a magnetic mode becomes unstable, their resulting bifurcation structure is different from that in the case of the NSE. But a common feature of the bifurcation properties of both systems (MHD and NSE) is that the dihedral group $D_{4}$ is a relevant subgroup, leaving invariant some secondary branches, as e.g. PER-3 in Table I.

Another interesting point in the comparison of both systems is the occurrence of a relaminarization window for the NSE, as reported in Refs. [8] and [9]. A second stable stationary solution, denoted by $\mathcal{A}_{2}$, different from the $\mathrm{ABC}$ flow, was found there. $\mathcal{A}_{2}$ coexists first with the stable $\mathrm{ABC}$ flow and then, after the $\mathrm{ABC}$ flow has become unstable, with time-dependent solutions, including chaotic ones, that have bifurcated from the ABC flow. For further increased $R, \mathcal{A}_{2}$ becomes attractive even for solutions with initial conditions close to the (unstable) ABC flow (called relaminarization). We must state here that we have not yet been able to find a second stable stationary branch for the MHD equations, yet we cannot exclude its existence, and numerical investigations with a much higher resolution using the pseudospectral method are going on.

\section{SPATIAL STRUCTURE OF THE MAGNETIC FIELD}

In this section we describe the structure of the magnetic field in real space and its changes under the influence of symmetry breaking bifurcations. In a kinematic dynamo study using the ABC flow with $A=B=C$, Galloway and Frisch [7] were the first to observe cigar-like concentrations of the magnetic field about velocity stagnation points. The $\mathrm{ABC}$ flow for the case $A=B=C$ has eight unstable stagnation points. The corresponding eigenvalues are real and have signs $(+,-,-)$ or $(-,+,+)$. The topological structure of the ABC flow, and in particular the intersections of the stable and unstable manifolds of the stagnation points, which form a complicated web of heteroclinic lines, were comprehensively discussed by Dombre et al. [4]. Stagnation points with a two-dimensional stable manifold have been denoted as of $\alpha$ type and those with a two-dimensional unstable manifold as of $\beta$ type. There are four stagnation points of each type and any two points of different type are connected by a straight line, forming a one-dimensional heteroclinic orbit. The cube diagonal through the points $(0,0,0)$ and $(2 \pi, 2 \pi, 2 \pi)$, for example, is a one-dimensional invariant manifold belonging 
to the stagnation points $(3 \pi / 4,3 \pi / 4,3 \pi / 4)$ ( $\beta$ type) and $(7 \pi / 4,7 \pi / 4,7 \pi / 4)$ ( $\alpha$ type). The rest of the ensemble of stagnation points and associated one-dimensional invariant manifolds may be obtained by applying the symmetry transformations $T_{2}, T_{3}, T_{4}$. The cigar-like structures of the magnetic field, observed by Galloway and Frisch [7] as well as by Galanti et al. [10] for the kinematic problem, are localized about the stagnation points of $\alpha$ type.

For the nonlinear problem, i.e., for the full MHD equations, one expects that after the first bifurcation, where the $\mathrm{ABC}$ flow loses its stability and a magnetic field appears, the structure of the fields differs at first only weakly from that of the kinematic problem. In the last section we already mentioned that this Hopf bifurcation retains the original symmetry for the newly created periodic branch Per 1 . This symmetry has also an essential influence on the spatial structure of the corresponding magnetic and velocity fields, here briefly discussed. A surprising feature of the new branch is that the eight stagnation points of the $\mathrm{ABC}$ flow survive, i.e., they remain time-independent zero-velocity points with the same location as for the original ABC flow. In Fig. 5 isosurfaces of the magnetic field strength for a level of $65 \%$ of the maximum value are drawn.

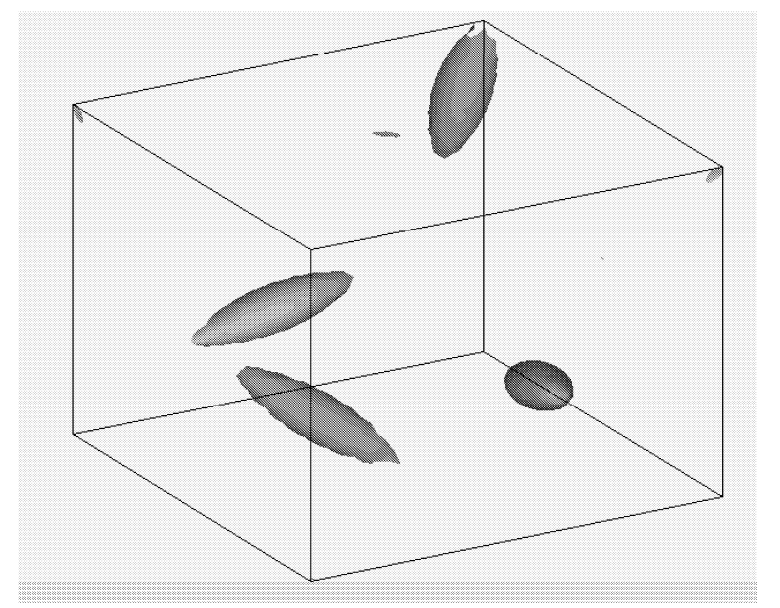

FIG. 5. Isosurfaces of the magnetic field with $65 \%$ of the maximal modulus for the symmetric branch $(\mathrm{R}=10)$ in the periodic box $(x, y, z$ between 0 and $2 \pi)$.

The cigar-like structure are clearly recognizable also in the nonlinear regime. Due to the temporal periodicity, the magnetic field oscillates and the shape of the isosurfaces depends also on the time of a snapshot, sometimes they look much more like blobs. To give an impression of the dynamics, we consider the fields on the diagonal line. As already mentioned, the stagnation points remain unchanged, but furthermore also their invariant one-dimensional manifolds are formed by the same straight lines. For the diagonal line, containing two stagnation points, this follows directly from the symmetry of the branch. Namely, as a consequence of the symmetry with respect to the cyclic group $Z_{3}$ as a subgroup of the full symmetry group, the velocity field has to be aligned with the diagonal direction. The same holds for the magnetic field.

In Fig. 6 the component of the magnetic field along the diagonal line is shown for different instants of a time period (stagnation points are marked by asterisks).

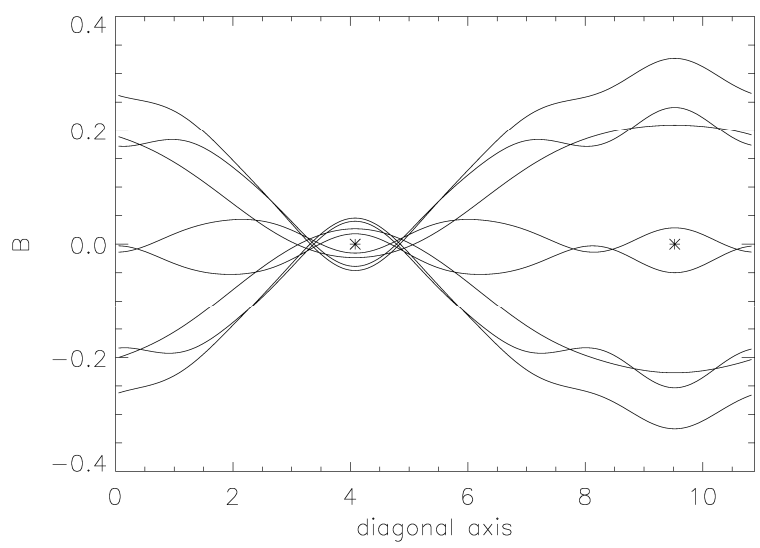

FIG. 6. Magnetic field component along the diagonal axis at different instants of time $(\mathrm{R}=10)$. Stagnation points are marked by asterisks.

Strong oscillations with a large amplitude occur around the stagnation point of $\alpha$ type, and the magnetic energy is mainly located in its neighborhood. The velocity field along the diagonal line oscillates only relatively weakly about the original $\mathrm{ABC}$ flow.

Fig. 7 shows a contour plot of the modulus of the magnetic field in a particular plane through the cube, $z=1 / 2(x+y)$, which contains also the (main) diagonal axis and its stagnation points. Thick lines correspond to levels above $65 \%$ of the maximum value and the cigar-like structure becomes again visible.

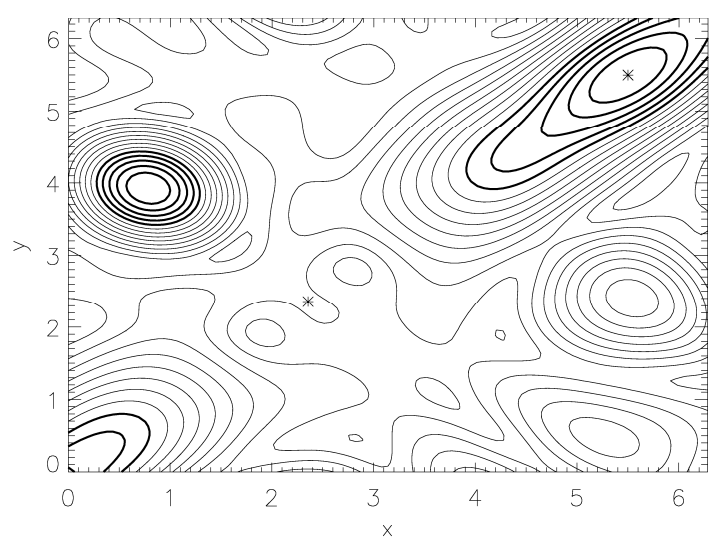


FIG. 7. Contour plot of the modulus of the magnetic field in the plane $z=1 / 2(x+y)(\mathrm{R}=10)$. The plane is parameterized by the coordinates $x$ and $y$. The stagnation points on the diagonal line are marked by asterisks. Thick lines correspond to contour levels above $65 \%$ of the maximum value.

For the asymmetric branch Per-4 only two of the stagnation points together with their invariant straight line survive. The others disappear, but they remain stagnation points for other branches obtained by symmetry transformations. The spatial structure of the magnetic field has also changed. For some instant of time just one blob-like structure located about the one $\alpha$ type stagnation point is visible. Then it is shrinking and the three other cigar-like field concentrations emerge, but without any stagnation points. This process and its reversal can be observed approximately two times within one period.

For the other asymmetric branch, Per-3, no stagnation points are present, but four cigar-like field concentrations may again be recognized. In contrast to the structures seen in Fig. 5, they are now deformed and shifted away from the location of the former stagnation points. The whole structure is oscillating and at some instants the magnetic energy seems to be distributed over the whole cube.

It is not the aim of this section to describe the structural properties of the magnetic field for all branches listed in Table I, but at last we would like to discuss briefly the chaotic branch Chaos-3. It is interesting that also for this branch, the magnetic energy is on average mainly concentrated in four tube-like structures. These oscillate and move irregularly through the cube. The appearance of just four strong-field regions is still reminiscent of the four $\alpha$ type stagnation points of the original $\mathrm{ABC}$ flow.

\section{DISCUSSION}

To test the sensivity of the results of our bifurcation analysis with respect to the degree of truncation in Fourier space, we partially used a much higher resolution by means of a pseudospectral method. The bifurcation structure seems to be the same or at least similar for all truncations. For instance, the instability of the primary $\mathrm{ABC}$ flow with respect to a periodic solution with non-vanishing magnetic field and the appearance of other coexisting periodic branches, denoted by Per-3 in Table I, together with their described symmetry properties, has been confirmed in high-resolution simulations. Quasiperiodic and periodic solutions have also been found applying the pseudospectral technique, but we have not yet been able to classify all solution branches so systematically as given in Table I. One of our further aims is to continue the bifurcation analysis for high resolutions, i.e., to determine the corresponding bifurcation diagram and to describe the symmetry breaking effects.
For the case of the (non-magnetic) NSE, Podvigina and Pouquet [9] have found a steady-state branch different from the $\mathrm{ABC}$ flow and coexisting with it (or with solutions bifurcating from it). The existence of a similar steady-state branches for the MHD equations remains an open problem, which we also plan to attack by means of high-resolution pseudospectral techniques.

\section{ACKNOWLEDGMENTS}

B. Galanti thanks the Max-Planck-Gesllschaft for its hospitality during his stay at the group of the University in Potsdam.

\section{APPENDIX}

Here we give a complete list of the symmetry transformations for which the MHD equations with the $\mathrm{ABC}$ forcing ( $A=B=C=R, \quad k_{0}=1$ ) given by Eqs. (1), $(17),(18)$ and (7) are equivariant (transformations of the local coordinates modulo $2 \pi$ ):

\section{$T_{1}:$ Identity}

$\begin{array}{lll}T_{2}: x_{1} \rightarrow x_{1}+\pi / 2, & x_{2} \rightarrow x_{3}-\pi / 2, & x_{3} \rightarrow-x_{2}+\pi / 2 \\ T_{3}: x_{1} \rightarrow x_{1}+\pi, & x_{2} \rightarrow-x_{2}, & x_{3} \rightarrow-x_{3}+\pi \\ T_{4}: x_{1} \rightarrow x_{1}-\pi / 2, & x_{2} \rightarrow-x_{3}+\pi / 2, & x_{3} \rightarrow x_{2}+\pi / 2 \\ T_{5}: x_{1} \rightarrow-x_{3}+\pi / 2, & x_{2} \rightarrow x_{2}+\pi / 2, & x_{3} \rightarrow x_{1}-\pi / 2 \\ T_{6}: x_{1} \rightarrow-x_{1}+\pi, & x_{2} \rightarrow x_{2}+\pi, & x_{3} \rightarrow-x_{3} \\ T_{7}: x_{1} \rightarrow x_{3}+\pi / 2, & x_{2} \rightarrow x_{2}-\pi / 2, & x_{3} \rightarrow-x_{1}+\pi / 2 \\ T_{8}: x_{1} \rightarrow x_{2}-\pi / 2, & x_{2} \rightarrow-x_{1}+\pi / 2, & x_{3} \rightarrow x_{3}+\pi / 2 \\ T_{9} ; x_{1} \rightarrow-x_{1}, & x_{2} \rightarrow-x_{2}+\pi, & x_{3} \rightarrow x_{3}+\pi \\ T_{10}: x_{1} \rightarrow-x_{2}+\pi / 2, & x_{2} \rightarrow x_{1}+\pi / 2, & x_{3} \rightarrow x_{3}-\pi / 2 \\ & & \\ T_{11}: x_{1} \rightarrow x_{3}+\pi, & x_{2} \rightarrow-x_{1}, & x_{3} \rightarrow-x_{2}+\pi \\ T_{12}: x_{1} \rightarrow-x_{2}, & x_{2} \rightarrow-x_{3}+\pi, & x_{3} \rightarrow x_{1}+\pi \\ T_{13}: x_{1} \rightarrow x_{2}, & x_{2} \rightarrow x_{3}, & x_{3} \rightarrow x_{1} \\ T_{14}: x_{1} \rightarrow x_{3}, & x_{2} \rightarrow x_{1}, & x_{3} \rightarrow-x_{1}+\pi \\ T_{15}: x_{1} \rightarrow x_{2}+\pi, & x_{2} \rightarrow-x_{3}, & x_{3} \rightarrow-x_{2} \\ T_{16}: x_{1} \rightarrow-x_{3}+\pi, & x_{2} \rightarrow x_{1}+\pi, & x_{3} \rightarrow-\pi \\ T_{17}: x_{1} \rightarrow-x_{3}, & x_{2} \rightarrow-x_{1}+\pi, & x_{3} \rightarrow x_{2}+\pi \\ T_{18}: x_{1} \rightarrow-x_{2}+\pi, & x_{2} \rightarrow x_{3}+\pi, & x_{3} \rightarrow-x_{1} \\ & & \end{array}$

In the list the transformations have been arranged such as to make visible the group structure of of the octahedral group $O$, the rotations which leave a cube invariant. It is known [17] that the group can be decomposed according 
to

$$
O=\dot{\cup}^{3} Z_{4} \dot{\cup}^{4} Z_{3} \dot{\cup}^{6} Z_{2}
$$

The elements $T_{2}, \ldots, T_{10}$ correspond to rotations by $90^{\circ}$ of a cube about the three axes through the middle points of its faces and form together with $T_{1}$ three copies of the cyclic group $Z_{4}$. The elements $T_{11}, \ldots, T_{18}$ may be interpreted as rotations by $120^{\circ}$ about the four diagonal axes (four copies of $Z_{3}$ ). Each element of the last block, finally, is together with $T_{1}$ isomorphic to $Z_{2}$ and is related to rotations by $180^{\circ}$ about the axes crossing opposite edges (all axes mentioned here also cross the middle point of the cube).

The the following Table VI is the group table for the octahedral group $O$, calculated from the transformations by means of the Mathematica Package [18].

[1] P. H. Roberts and A. M. Soward, Ann. Rev. Fluid Mech. 24, 459 (1992).

[2] V. I. Arnold, C. R. Acad. Sci. Paris 261, 17 (1965).

[3] V. I. Arnold and E. I. Korkina, Vest. Mosk. Univ. Mat. Mekh. 3, 43 (1983).

[4] T. Dombre et al., J. Fluid Mech. 167, 353 (1986).

[5] D. Galloway and U. Frisch, Geophys. Astrophys. Fluid Dyn. 36, 53 (1986).

[6] B. Galanti, A. Pouquet, and P. L. Sulem, in Theory of Solar and Planetary Dynamos, edited by M. R. E. Proctor, P. C. Matthews, and A. M. Rucklidge (Cambridge University Press, Cambridge, 1993), pp. 99-103.

[7] D. Galloway and U. Frisch, J. Fluid Mech. 180, 557 (1987).

[8] O. Zheligovsky and A. Pouquet, in Theory of Solar and Planetary Dynamos, edited by M. R. E. Proctor, P. C. Matthews, and A. M. Rucklidge (Cambridge University Press, Cambridge, 1993), pp. 347-354.

[9] O. Podvigina and A. Pouquet, Physica D 75, 471 (1994).

[10] B. Galanti, P. L. Sulem, and A. Pouquet, Geophys. Astrophys. Fluid Dyn. 66, 183 (1992).

[11] F. Feudel, N. Seehafer, and O. Schmidtmann, Phys. Lett. A 202, 73 (1995).

[12] P. H. Roberts, An Introduction to Magnetohydrodynamics (Longmans, London, 1967).

[13] V. I. Arnold, in Some Problems of Modern Analysis, edited by V. M. Tikhomirov (Moscow State University, Moscow, 1984), pp. 8-21.

[14] I. Shimada and T. Nagashima, Progr. Theor. Phys. 61, 1605 (1979).

[15] F. Feudel, N. Seehafer, and O. Schmidtmann, Mathematics and Computers in Simulation 40, (to appear 1996).

[16] A. Wirth, S. Gama, and U. Frisch, J. Fluid Mech. 288, 249 (1995).

[17] M. Golubitsky, I. Stewart, and D. G. Schaeffer, Singularities and Groups in Bifurcation Theory (Springer-Verlag, New York, 1988), Vol. II.
[18] S. Wolfram, Mathematica (Addison-Wesley, Redwood City, 1988). 


\begin{tabular}{|c|c|c|c|c|c|c|c|c|c|c|c|c|c|c|c|c|c|c|c|c|c|c|c|c|}
\hline & $T_{1}$ & 2 & $T_{3}$ & $T_{4}$ & $T_{5}$ & $T_{6}$ & $T_{7}$ & 8 & 9 & 10 & $T_{11}$ & $T_{12}$ & $T_{13}$ & 14 & $T_{15}$ & $T_{16}$ & $T_{17}$ & $T_{18}$ & $T_{19}$ & $T_{20}$ & 21 & 22 & 23 & 12 \\
\hline & $T_{1}$ & $T_{2}$ & $\Gamma$ & $\pi$ & $T$ & $T_{6}$ & $T_{7}$ & $T$ & $T_{9}$ & $T_{10}$ & & & & 4 & $T_{15}$ & $T_{16}$ & $T_{17}$ & $T_{18}$ & & $T_{20}$ & 1 & 22 & & $T$ \\
\hline  & $T_{2}$ & $T_{3}$ & $T_{1}$ & $T_{1}$ & $T_{16}$ & $\Gamma_{20}$ & $T_{11}$ & $T_{13}$ & $T_{22}$ & $T_{18}$ & $T_{23}$ & $T_{10}$ & $T_{19}$ & $T_{7}$ & $T_{0}$ & $T_{24}$ & $T_{5}$ & $T_{21}$ & $T_{15}$ & $T_{9}$ & 12 & $T_{6}$ & 14 & $T_{1}$ \\
\hline & $T_{3}$ & $T_{4}$ & $T_{1}$ & $T$ & & $T_{9}$ & $T_{23}$ & $T_{19}$ & $\Gamma$ & $T_{21}$ & $T_{14}$ & $T_{18}$ & $T_{15}$ & $T_{11}$ & $T_{13}$ & $T_{17}$ & $T_{16}$ & $T_{12}$ & T & $T_{22}$ & 10 & $T_{20}$ & 7 & $T_{5}$ \\
\hline 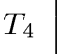 & $T_{4}$ & 7 & T & $T$ & & $T_{2}$ & $T_{14}$ & $T_{15}$ & $T_{20}$ & $T_{12}$ & $T$ & & $T_{8}$ & & & $T$ & $T_{24}$ & $T_{10}$ & $T_{13}$ & $T_{0}$ & $\Gamma_{18}$ & $T_{9}$ & 1 & $T_{1}$ \\
\hline$\Gamma_{5}$ & $T_{5}$ & $T_{13}$ & $T_{23}$ & $T_{12}$ & $T_{6}$ & $T_{7}$ & $T_{1}$ & $T_{17}$ & $T_{24}$ & $T_{16}$ & $T_{8}$ & $T_{20}$ & $T_{22}$ & $T_{10}$ & $T_{4}$ & $T_{19}$ & $T_{21}$ & $T_{2}$ & $T_{14}$ & $T_{15}$ & 11 & $T_{18}$ & 9 & $\Gamma_{3}$ \\
\hline$\Gamma_{6}$ & $T_{6}$ & $T_{22}$ & $T_{9}$ & $T_{20}$ & $T_{7}$ & $T_{1}$ & $T_{5}$ & $T_{21}$ & $T_{3}$ & $T_{19}$ & $T_{17}$ & $T_{15}$ & $T_{18}$ & $T_{16}$ & $T_{12}$ & $T_{14}$ & $T_{11}$ & $T_{13}$ & $T_{10}$ & 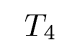 & $\Gamma_{8}$ & $T_{2}$ & 24 & $T_{2}$ \\
\hline$\Gamma_{7}$ & $T_{7}$ & $T_{18}$ & $T_{24}$ & $T_{15}$ & $T_{1}$ & $T_{5}$ & $T_{6}$ & $T_{11}$ & $T_{23}$ & $T_{14}$ & $T_{21}$ & $T_{4}$ & $T$ & $T_{19}$ & $T_{20}$ & $T_{10}$ & $T_{8}$ & $T_{22}$ & $T_{16}$ & $T_{12}$ & 17 & $\Gamma_{13}$ & 3 & $T_{9}$ \\
\hline$\Gamma$ & $T_{8}$ & $T_{11}$ & $T_{21}$ & & $T_{13}$ & $T_{19}$ & $T_{15}$ & $T_{9}$ & $T_{10}$ & $T_{1}$ & $T_{20}$ & $T_{5}$ & $T_{23}$ & & $T_{24}$ & $T_{2}$ & $T_{22}$ & & & $\Gamma_{16}$ & 6 & 14 & 2 & $T_{18}$ \\
\hline$\Gamma$ & $T_{9}$ & $I$ & $T_{6}$ & & & $T$ & $T_{2}$ & $T_{\perp}$ & $t$ & $T_{8}$ & $T_{16}$ & $T_{13}$ & & $T_{17}$ & $T_{18}$ & $T_{11}$ & $T_{14}$ & $T_{15}$ & & 2 & 9 & 4 & $T_{5}$ & $T_{7}$ \\
\hline  & $T_{10}$ & $T_{16}$ & $T_{19}$ & $T_{14}$ & $T_{12}$ & $T_{21}$ & $T_{18}$ & $T_{1}$ & $T$ & $T$ & $T_{0}$ & $T_{23}$ & $T_{-}$ & $T_{22}$ & $T_{7}$ & $T_{0}$ & $T$ & $T_{24}$ & $T$ & $T_{11}$ & $\Gamma_{0}$ & $T_{17}$ & 13 & $T_{15}$ \\
\hline 11 & $T_{11}$ & $T_{21}$ & $T_{17}$ & $T$ & $T_{2}$ & $T_{16}$ & $T_{20}$ & $T_{23}$ & $T_{14}$ & $T_{7}$ & $T_{12}$ & $T$ & $T$ & $T_{15}$ & $T_{9}$ & $T_{18}$ & $T_{13}$ & $T_{\mathrm{s}}$ & $T_{24}$ & $T_{10}$ & $T_{-}$ & 19 & $\Gamma_{4}$ & $T_{2}$ \\
\hline$T_{12}$ & $T_{12}$ & $T_{5}$ & $T_{13}$ & $T_{23}$ & $T_{21}$ & $T_{18}$ & $T_{10}$ & $T_{4}$ & $T_{15}$ & $T_{20}$ & $T_{1}$ & $T_{11}$ & $T_{17}$ & $T_{9}$ & $T_{14}$ & $T_{c}$ & $T_{3}$ & $T_{16}$ & $T_{22}$ & $T_{7}$ & $T_{2}$ & $\Gamma_{24}$ & 8 & $T_{1}$ \\
\hline 13 & $T_{13}$ & $T_{23}$ & $T_{12}$ & $T_{-}$ & $T_{19}$ & $T_{15}$ & $T_{8}$ & $T_{20}$ & $T_{18}$ & $T$ & $T$ & $T_{16}$ & $T_{14}$ & $T$ & $T_{17}$ & 7 & 7 & $\Gamma 11$ & & $T_{24}$ & $T_{20}$ & $T_{7}$ & 10 & $T_{21}$ \\
\hline 74 & $T_{14}$ & $T_{10}$ & $T_{16}$ & & $T$ & $T_{17}$ & $T_{22}$ & $T_{7}$ & $T_{11}$ & $T_{23}$ & $T_{18}$ & $T$ & 1 & & $I$ & $T_{12}$ & $T_{15}$ & & $T_{5}$ & $T_{21}$ & 24 & $T_{8}$ & 2 & $T_{2}$ \\
\hline$\Gamma 15$ & $T_{15}$ & $T_{7}$ & $T_{18}$ & T & 7 & $T_{13}$ & $T_{19}$ & $T_{20}$ & $T_{12}$ & $T_{4}$ & $T_{6}$ & $T_{17}$ & $T_{11}$ & 7 & 6 & 1 & & 4 & & $T_{5}$ & 22 & 23 & 21 & $\Gamma_{10}$ \\
\hline$T_{16}$ & $T_{16}$ & $T_{19}$ & $T_{14}$ & $T_{10}$ & $T_{20}$ & $T_{11}$ & $T_{2}$ & $T_{5}$ & $T_{17}$ & $T_{24}$ & $T_{13}$ & $T_{9}$ & $T_{6}$ & $T_{18}$ & $T_{1}$ & 15 & $\Gamma_{12}$ & $T_{3}$ & $T_{7}$ & 8 & $T_{23}$ & 21 & & $T_{4}$ \\
\hline$\Gamma 17$ & $T_{17}$ & $T_{8}$ & $T_{11}$ & $T_{21}$ & $T_{22}$ & $T_{14}$ & $T_{4}$ & $T_{24}$ & $T_{16}$ & $T_{5}$ & $T_{15}$ & $T$ & $T$ & $T_{12}$ & $T_{3}$ & $T_{13}$ & $T_{18}$ & $T_{1}$ & 3 & $T_{19}$ & $T_{7}$ & 10 & & $T_{2}$ \\
\hline$\Gamma_{18}$ & $T_{18}$ & $T_{24}$ & $T_{15}$ & $T_{-}$ & T & 12 & $T_{0}$ & $T_{2}$ & $T_{13}$ & $T_{22}$ & $T$ & $T_{14}$ & $T$ & $T$ & 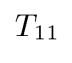 & $T_{9}$ & 7 & 17 & 0 & 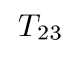 & $\Gamma_{1}$ & $\Gamma_{5}$ & 9 & \\
\hline 19 & $T_{19}$ & $T_{14}$ & $T_{10}$ & $T_{16}$ & $T$ & $T_{8}$ & $T_{13}$ & $T_{6}$ & $T_{21}$ & $T_{3}$ & $T_{22}$ & $T_{24}$ & 7 & 7 & $T_{5}$ & 7 & $T_{20}$ & 3 & & $T_{17}$ & $T_{9}$ & $T_{11}$ & 18 & $T_{1}$ \\
\hline$T_{20}$ & $T_{20}$ & $T_{6}$ & $T_{22}$ & $T_{9}$ & $T_{11}$ & $T_{2}$ & $T_{16}$ & $T_{12}$ & $T_{4}$ & $T_{15}$ & $T_{5}$ & $T_{8}$ & $T_{21}$ & $T_{24}$ & 10 & $T_{7}$ & $T_{23}$ & $T_{19}$ & $T_{18}$ & 1 & $T_{13}$ & $T_{3}$ & 17 & $T_{1}$ \\
\hline$T_{21}$ & $T_{21}$ & $T_{17}$ & $T_{8}$ & $T_{11}$ & $T_{18}$ & $T_{10}$ & $T_{12}$ & $T_{3}$ & $T_{19}$ & $T_{6}$ & 4 & 17 & $T_{24}$ & $T_{20}$ & $T_{23}$ & 22 & 2 & $T_{5}$ & & $T_{14}$ & 1 & $T_{16}$ & 15 & $I_{1}$ \\
\hline$T_{22}$ & $T_{22}$ & $T_{9}$ & $T_{20}$ & $T_{6}$ & $T_{14}$ & $T_{4}$ & $T_{17}$ & $T_{18}$ & $T_{2}$ & $T_{13}$ & $T_{24}$ & $T_{19}$ & $T_{10}$ & $T_{5}$ & $T_{21}$ & $T_{23}$ & 17 & 8 & 112 & 3 & 15 & $T_{1}$ & 16 & 1 \\
\hline$T_{23}$ & $T_{23}$ & $T_{12}$ & $T_{5}$ & $T_{13}$ & $T_{3}$ & $I_{24}$ & $T_{9}$ & $T_{14}$ & $T_{7}$ & $T_{11}$ & $T_{10}$ & 12 & 14 & 18 & $T_{22}$ & $T_{21}$ & $T_{19}$ & $T_{20}$ & $T_{17}$ & $T_{18}$ & $T_{16}$ & $T_{15}$ & 1 & \\
\hline$\Gamma_{24}$ & $T_{24}$ & $T_{15}$ & 7 & $T_{18}$ & $T_{9}$ & 23 & $T_{3}$ & 116 & $T_{5}$ & $T_{17}$ & $T_{19}$ & $T_{22}$ & $T_{20}$ & 1 & $T_{2}$ & $T_{8}$ & 0 & $T_{4}$ & T & $T_{13}$ & $T_{14}$ & $T_{12}$ & 6 & \\
\hline
\end{tabular}

